Note

\title{
Isolation of Indole-3-Aldehyde As a Growth Inhibitor of Legionella pneumophila from Diaion HP-20 Resins Used to Culture the Bacteria
}

\author{
HIROAKI INOUE ${ }^{*}$, YOUSUKE YAJIMA², GENJI KAWANO', \\ HIROMICHI NAGASAWA ${ }^{2}$ AND SHOHEI SAKUDA ${ }^{2}$
}

\author{
${ }^{1} T s u k u b a$ Research Laboratories, Aquas Corporation, 4-4 Midorigahara, Tsukuba, Ibaraki 300-2646, \\ and ${ }^{2}$ Department of Applied Biological Chemistry, Graduate School of Agricultural and Life Sciences, \\ The University of Tokyo, Bunkyo-ku, Tokyo 113-8657, Japan
}

Received 5 December 2003/Accepted 26 January 2004

\begin{abstract}
The addition of Diaion HP-20 resin to a medium can enhance the growth of the Legionella species. We recently reported that elemental sulfur was isolated as a growth-inhibiting substance produced endogenously by Legionella pneumophila from the methanol extracts of the resins used to culture the bacteria. In this study, we attempted to isolate other growthinhibiting substances involved in the extracts, and identified indole-3-aldehyde as one of the inhibitors. The compound was involved in the medium as a constituent and it weakly inhibited the growth of $L$. pneumophila, indicating that the compound partly contributes to inhibit the bacterial growth when cultured without HP-20 resins.
\end{abstract}

Key words : Legionella/Indole/Growth inhibitor.

Legionella pneumophila is a gram-negative bacteria ubiquitously found in natural and man-made water systems. If people inhaled aerosolized water from sources contaminated with L. pneumophila, they could come down with a severe form of pneumonia called Legionnaires' disease (Vogel and Isberg, 1999). Since the mortality from the pneumonia can approach 30 to 40 without prompt treatment with effective antibiotics, it is very important to control and manage Legionella contamination in the water systems.

L. pneumophila requires a specialized medium for growth (Pine et al., 1979). The medium contains iron (III) and L-cysteine as essential components. In addition to these nutrients, activated charcoal is usually added to the medium since it can strongly enhance the bacterial growth. Since charcoal is a good absorbent, its role during the culture process is thought to be the absorption and detoxification of a toxic sub-

${ }^{*}$ Corresponding author. Tel: +81-29-847-6000, Fax : + 81-29-847-6080 stance, which may inhibit the bacterial growth (Hoffman et al., 1983; Pine et al., 1979; Ristroph et al., 1980). If the substance has high specificity toward Legionella growth, it may be very useful as a lead compound for developing effective drugs to prevent Legionella contamination in water systems. From this viewpoint, we focused on the substance. We developed a new culture method, in which Diaion HP-20 resin was used as an absorbent instead of activated charcoal. In the case of charcoal, it is not easy to obtain only charcoal particles from the mixture of charcoal and bacterial cells involved in the broth. Diaion HP-20 resin is a synthetic absorbent of cross-linked polystyrene, and spherical particles of the resin with diameters of $0.5 \mathrm{~mm}$ can be easily recovered by filtration from the culture broth without contamination of the bacterial cells. We recently reported that elemental sulfur was isolated from methanol extracts of Diaion HP-20 resins used to culture the bacteria as a main growth-inhibiting substance produced endogenously by L. pneumophila (Inoue et al., 2002). Since some active fractions other than that containing 
elemental sulfur were obtained during the purification of elemental sulfur from the methanol extracts, we have been continuing to isolate of other active substances from the extracts. In this paper, we describe the isolation of indole-3-aldehyde as one of the growth inhibitors, which is shown to be involved as a medium constituent.

Cultivation of Legionella pneumophila GIFU9134 was performed with a medium $(0.1 \%, w / v, \alpha$-ketoglutaric acid, $0.5 \%, \mathrm{w} / \mathrm{v}, \mathrm{K}_{2} \mathrm{HPO}_{4}, 1.5 \%$, w/v, yeast extract, $0.3 \%, \mathrm{w} / \mathrm{v}, \mathrm{NaCl}, 0.1 \%, \mathrm{w} / \mathrm{v}, \mathrm{L}$-cysteine hydrochloride, $0.05 \%, w / v$, ferric pyrophosphate, $\mathrm{pH}$ 6.8) containing Diaion HP-20 resin (5\%) according to the procedure previously reported (Inoue et al., 2002). After 7 days' cultivation at $37{ }^{\circ} \mathrm{C}$ with shaking at $120 \mathrm{rpm}$, the culture broth obtained $(1,000 \mathrm{ml} \times$ 20) was put into a column with a cotton plug to separate the Diaion HP-20 resin. After being washed with water (5 liters), the column with the resin was eluted with methanol (3 liters). This operation was repeated four more times to obtain the methanol solution from 100 liters of culture broth. The solution was concentrated, and extracted with ethyl acetate (EtOAc). After being washed with saturated $\mathrm{NaHCO}_{3}$ solution, the EtOAc solution was concentrated, and the obtained residue $(3.24 \mathrm{~g})$ was applied to a silica gel column (Wakogel C-200, $52 \times 150 \mathrm{~mm}$ ). The column was eluted stepwise with 1.5 liters each of $n$-hexane, $n$-hexane/EtOAc (90/10), $n$-hexane/EtOAc (80/ 20), $n$-hexane/EtOAc (70/30), n-hexane/EtOAc (60/40), $n$-hexane/EtOAc (50/50) and EtOAc. As described previously, crystals of elemental sulfur (20.3 mg) were obtained as the main active component from the $n$-hexane fraction. To search for other active substances, growth inhibitory activity toward $L$. pneumophila of other fractions from the column was tested by the broth dilution method as follows. Samples were dissolved in ethanol or dimethyl sulfoxide at appropriate concentrations, and the solution $(5 \mu \mathrm{l})$ was mixed with a broth of $L$. pneumophila (995 $\mu \mathrm{l}, 10^{4}$ cells). After 48 hours of incubation, growth of the bacteria was judged from the turbidity of the broth. By this method, $n$-hexane/EtOAc (60/40) and (50/50) fractions were shown to have growth inhibitory activity with a minimum inhibitory concentration (MIC) value of around $25 \mu \mathrm{g} / \mathrm{ml}$.

We could not isolate an active component from the $n$-hexane/EtOAc (50/50) fraction since the activity was divided into many fractions without an increase in the relative activity by the usually employed method such as silica gel column chromatography or reverse-phase high performance liquid chromatography (HPLC). The same problem was also observed in the case of purification of the $n$-hexane/EtOAc
(60/40) fraction. However, one of the active fractions $(6.7 \mathrm{mg}$ ) was obtained by Sephadex LH-20 column chromatography $(23 \times 490 \mathrm{~mm}$, methanol $)$ of the $n$ hexane/EtOAc (60/40) fraction, and further purification of the fraction by using a reverse-phase HPLC (Capcell-Pak $\mathrm{C}_{18} 10 \times 250 \mathrm{~mm}, 0-50 \%$ liner gradient with acetonitrile/water) afforded an active substance $(650 \mu \mathrm{g})$, which was identified as indole-3-aldehyde (Fig. 1) by its spectral data (FAB-MS (glycerol matrix) $\mathrm{m} / \mathrm{z} 145(\mathrm{M}+\mathrm{H})^{+}$; ${ }^{1} \mathrm{H} \mathrm{NMR}(500 \mathrm{MHz}, \mathrm{MeOH}-$ $\left.d_{6}\right) \delta 9.86(1 \mathrm{H}, \mathrm{q}, 8-\mathrm{H}), 8.16(1 \mathrm{H}, \mathrm{d}, J=8.0 \mathrm{~Hz}, 7-\mathrm{H})$, $8.10(1 \mathrm{H}, \mathrm{s}, 2-\mathrm{H}), 7.48(1 \mathrm{H}, \mathrm{d}, J=8.0 \mathrm{~Hz}, 4-\mathrm{H}), 7.28$ $(1 \mathrm{H}, \mathrm{dt}, J=1.0,8.0 \mathrm{~Hz}, 6-\mathrm{H}), 7.42(1 \mathrm{H}, \mathrm{dt}, J=1.0,8.0$ $\mathrm{Hz}, 5-\mathrm{H}) ;{ }^{13} \mathrm{C}$ NMR $\left(125 \mathrm{MHz}, \mathrm{MeOH}-d_{6}\right) \delta 187.5$ (C8), 139.9 (C-2), 139.1 (C-7a), 125.9 (C-3a), 125.2 (C-6), 123.8 (C-5), 122.6 (C-4), 120.3 (C-3), 113.3 (C-7).).

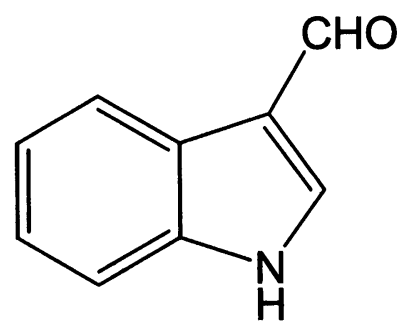

FIG. 1. Structure of Indole-3-aldehyde.

Table 1 shows the growth inhibitory activity of indole-3-aldehyde and its related indole derivatives against $L$. pneumophila tested by the method mentioned above. Indole-3-aldehyde showed weak activity with a MIC value of $100 \mu \mathrm{g} / \mathrm{ml}$. Among the listed compounds, the activity of indole-3-acetic acid, indole-3-propionic acid and indole-3-butyric acid has already been reported by Grossowicz (Grossowicz, 1990). He found the effects of these compounds during a study on amino acids involved in a medium for the bacterial growth, and showed indole-3-propionic

TABLE 1. Antimicrobial activity of indole compounds against $L$. pneumophila.

\begin{tabular}{lc}
\hline \multicolumn{1}{c}{ Compound } & MIC $(\mu \mathrm{g} / \mathrm{ml})$ \\
\hline Indole-3-aldehyde & 100 \\
Indole-3-ethanol & $>200$ \\
Indole-3-propanol & 200 \\
Indole-3-butanol & 100 \\
Indole-3-carboxylic acid & $>200$ \\
Indole-3-acetic acid & 200 \\
Indole-3-propionic acid & 12.5 \\
Indole-3-butyric acid & 100 \\
Indole-3-acrylic acid & 50 \\
Indole-3-lactic acid & $>200$ \\
L-Tryptophan & $>200$ \\
\hline
\end{tabular}


acid with the strongest activity among the three acids has specific activity against the Legionella species. This time, we showed that indole-3-aldehyde and some indole alcohols also have antimicrobial activity against $L$. pneumophila.

To clarify whether indole-3-aldehyde is a metabolite of $L$. pneumophila or a medium constituent, we analyzed the methanol extracts of Diaion HP-20 resins recovered from the medium without inoculation of the bacteria. Two samples were prepared from the resins recovered from the medium before and after autoclaving. Each extract was purified by EtOAc extraction and reverse-phase HPLC to obtain an indole3-aldehyde sample. The purity of each sample was confirmed by NMR analysis, and the amount of indole-3-aldehyde involved in each sample was quantified from the peak area on HPLC. As a result, it was shown that the contents of indole-3-aldehyde in the extracts prepared before and after autoclaving were 17 and $46 \mu \mathrm{g} /$ liter, respectively. This indicated that indole-3-aldehyde was involved in the medium as a constituent and that it was increased by autoclaving.

The methanol extracts of Diaion HP-20 resins used to culture L. pneumophila show strong growthinhibiting activity toward the bacteria itself. We previously reported that the amount of elemental sulfur involved in the extracts is enough to inhibit the bacterial growth if it is present in solution (Inoue et al., 2002). In this study, we found that the total growthinhibiting activity of the $n$-hexane/EtOAc (60/40) and (50/50) fractions obtained from the silica gel column for purification of active components other than the elemental sulfur may also be as strong as to affect the bacterial growth, which is estimated from the MIC value $(25 \mu \mathrm{g} / \mathrm{ml})$ and the yields $(60 / 40,129$ $\mathrm{mg} ; 50 / 50,31.2 \mathrm{mg}$ ). An active compound with strong activity was not obtained from the fractions, but we identified indole-3-aldehyde as one of the growth inhibitors involved in the fractions. The yield and activity of indole-3-aldehyde showed that its contribution to inhibit the bacterial growth may be small, but we first showed its activity against $L$. pneumophila. Studies to clarify the overall aspects of the active compounds involved in the Diaion HP-20 resins used to culture $L$. pneumophila and their role in the metabolism of the bacteria during growth are now in progress.

\section{ACKNOWLEDGEMENTS}

We thank Akiko Noda, Tomoyuki Iwasawa and Yuuji Saruwatari for their technical support and helpful comments. This work was supported by a Grant-in-Aid for Scientific Research (No. 12556016) from the Ministry of Education, Culture, Sports, Science, and Technology of Japan.

\section{REFERENCES}

Grossowicz, N. (1990) Phytohormones as specific inhibitors of Legionella pneumophila growth. /sr. J. Med. Sci., 26, 184-190.

Hoffman, P. S., Pine, L., and Bell, S. (1983) Production of superoxide and hydrogen peroxide in medium used to culture Legionella pneumophila: catalytic decomposition by charcoal. Appl. Environ. Microbiol., 45, 784-791.

Inoue, H., Kawano, G., Nagasawa, H., and Sakuda, S. (2002) Isolation of elemental sulfur as a self-growthinhibiting substance produced by Legionella pneumophila. Appl. Environ. Microbiol., 68, 4809-4811.

Pine, L., George, J. R., Reeves, M. W., and Harrell, W. K. (1979) Development of chemically defined liquid medium for growth of Legionella pneumophila. J. Clin. Microbiol., 9, 615-626.

Ristroph, J. D., Hedlund, K. W., and Allen, R. G. (1980) Liquid medium for growth of Legionella pneumophila. J. Clin. Microbiol., 11, 19-21.

Vogel, J. P., and Isberg, R. R. (1999) Cell biology of Legionella pneumophila. Curr. Opin. Microbiol., 2, 30-40. 